\title{
Ferramentas gerenciais para organização da linha do cuidado do acidente vascular cerebral: uma revisão integrativa
}

\author{
Management tools protocols for organizing the cerebral accident care line: an integrative review \\ Herramientas de gestión para organizar la línea de cuidado de accidentes cerebrales: una revisión \\ integrativa
}

Recebido: 18/05/2021 | Revisado: 28/05/2021 | Aceito: 31/05/2021 | Publicado: 17/06/2021

\author{
Cláudia Heidtmann Dias \\ ORCID: https://orcid.org/0000-0002-4118-8551 \\ Fundação Santa Casa de Misericórdia, Brasil \\ E-mail: clauheidtmann@gmail.com \\ Pilar Maria de Oliveira Moraes \\ ORCID: https://orcid.org/0000-0003-2817-4574 \\ Fundação Santa Casa de Misericórdia, Brasil \\ E-mail: pilarmoraesnutri@gmail.com \\ Xaene Maria Fernandes Duarte Mendonça \\ ORCID: https://orcid.org/0000-0002-0958-276X \\ Fundação Santa Casa de Misericórdia, Brasil \\ E-mail: xaene@ufpa.br \\ Thaís de Oliveira Carvalho Granado Santos \\ ORCID: https://orcid.org/0000-0001-9496-4561 \\ Fundação Santa Casa de Misericórdia, Brasil \\ E-mail: thaisgranadosantos@gmail.com \\ Patrícia Socorro Coelho Portal \\ ORCID: https://orcid.org/0000-0002-0080-6675 \\ Fundação Santa Casa de Misericórdia, Brasil \\ E-mail: patricia.portal@yahoo.com.br \\ Ilma Pastana Ferreira \\ ORCID: https://orcid.org/0000-0002-9152-3872 \\ Fundação Santa Casa de Misericórdia, Brasil \\ E-mail: ilma.pastana@uepa.br \\ José Augusto Carvalho de Araújo \\ ORCID: https://orcid.org/0000-0002-4503-7857 \\ Universidade do Estado do Pará, Brasil \\ E-mail: augustocarvalho@uepa.br \\ Salma Brito Saraty \\ ORCID: https://orcid.org/0000-0002-3048-3985 \\ Fundação Santa Casa de Misericórdia, Brasil \\ E-mail: salmasaratybel@hotmail.com
}

\begin{abstract}
Resumo
Objetivo: Realizar revisão integrativa da literatura acerca das ferramentas gerenciais utilizadas na Linha do Cuidado do Acidente Vascular Cerebral. Metodologia: Para seleção dos artigos, utilizou-se as bases de dados: LILACS (Literatura Latino-Americana em Ciências da Saúde), MEDLINE (National Library of Medicine), SCOPUS - Base de dados multidisciplinar e CINAHL (Cumulative Index of Nursing and Allied Health Literature), e idiomas Inglês, Espanhol e Português. A pesquisa ocorreu de novembro de 2020 a abril de 2021, utilizando os descritores controlados em Inglês "Management Decisions" e "stroke", mediados pelo operador booleano "and", visando ampliar o quantitativo de estudos. Os descritores foram extraídos do DECS (Descritores em Ciências da Saúde) do Portal BVS, do MESH (Medical Subject Headings) da National Library, e Portal de Periódicos CAPES/ MEC. Resultados: As tecnologias de educação foram as de maior achado na literatura comparadas com as tecnologias inovadoras, como: panfletos, cartilhas e palestras, mostrando relevância para o enfrentamento do paciente e a comunidade contribuindo com a identificação precoce dos primeiros sinais e sintomas, o que ainda pode ser considerado um desafio neste trabalho. Conclusão: É possível melhorar a qualidade de vida dos pacientes acometidos de AVC por meio da existência de ferramentas de tecnologias gerenciais que garantam de forma rápida e efetiva a comunicação entre a equipe multidisciplinar e a gerência dos serviços de saúde.
\end{abstract}

Palavras-chave: Acidente vascular cerebral; Comunicação em saúde; Gestão da qualidade; Decisões gerenciais. 


\begin{abstract}
Objective: Conduct an integrative literature review about the management tools used in the Stroke Care Line. Methodology: For the articles selection, the following databases were used: LILACS (Latin American Literature in Health Sciences), MEDLINE (National Library of Medicine), SCOPUS - Multidisciplinary database and CINAHL (Cumulative Index of Nursing and Allied Health Literature), in English, Spanish and Portuguese. The research took place from November 2020 to April 2021, using the controlled descriptors in English "Management Decisions" and "stroke", mediated by the Boolean operator "and", aiming to expand the number of studies. The descriptors were extracted from the DECS (Health Sciences Descriptors) of the BVS Portal, the MESH (Medical Subject Headings) of the National Library, and the CAPES / MEC Journal Portal. Results: Education technologies were the most found in the literature compared to innovative technologies, such as: pamphlets, booklets and lectures, showing relevance for facing the patient and the community, contributing to the early identification of the first signs and symptoms, which can still be considered a challenge in this work. Conclusion: It is possible to improve the quality of life of stroke patients through the existence of managerial technology tools that guarantee a fast and effective communication between the multidisciplinary team and the management of health services.
\end{abstract}

Keywords: Stroke; Health communication; Quality management; Management decisions.

\title{
Resumen
}

Objetivo: Realizar una revisión integradora de la literatura sobre las herramientas de manejo utilizadas en la Línea de atención al ictus. Metodología: Para la selección de artículos se utilizaron las siguientes bases de datos: LILACS (Literatura Latinoamericana en Ciencias de la Salud), MEDLINE (Biblioteca Nacional de Medicina), SCOPUS - Base de datos multidisciplinaria y CINAHL (Índice acumulativo de literatura en enfermería y afines en salud), y Inglés, español y portugués. La investigación se llevó a cabo desde noviembre de 2020 hasta abril de 2021, utilizando los descriptores controlados en inglés "Management Decisions" y "stroke", mediados por el operador booleano "and", con el objetivo de ampliar el número de estudios. Los descriptores fueron extraídos de los DECS (Descriptores de Ciencias de la Salud) del Portal BVS, los MESH (Encabezamientos de Materia Médica) de la Biblioteca Nacional y el Portal de Revistas CAPES / MEC. Resultados: Las tecnologías educativas fueron las más encontradas en la literatura en comparación con las tecnologías innovadoras, tales como: panfletos, folletos y conferencias, mostrando relevancia para el enfrentamiento del paciente y la comunidad, contribuyendo a la identificación temprana de los primeros signos y síntomas, lo que todavía puede considerarse un desafío en este trabajo. Conclusión: Es posible mejorar la calidad de vida de los pacientes con ictus mediante la existencia de herramientas tecnológicas de gestión que garanticen una comunicación rápida y eficaz entre el equipo multidisciplinario y la dirección de los servicios de salud.

Palabras-clave: Accidente vascular cerebral; Comunicación sanitaria; Gestión de la calidad; Las decisiones de gestión.

\section{Introdução}

O Acidente Vascular Cerebral (AVC) é considerado a principal causa de mortalidade no Brasil e no mundo, além de ser também responsável por um grande número de internações hospitalares em adultos e idosos. Além da mortalidade, outra medida igualmente importante do impacto do AVC são as sequelas neurológicas, pois a grande maioria das pacientes vítimas da doença desenvolve deficiência completa ou parcial (Maniva et al., 2018).

$\mathrm{O}$ estudo em questão mostra quais as principais ferramentas gerenciais utilizadas por equipes multiprofissionais de saúde, que realizam assistência aos pacientes acometidos por doenças cerebrovasculares e, a importância da gestão da qualidade, somadas às tecnologias de informação para o alcance de melhor atendimento dos usuários acometidos por doença cerebrovasculares do Sistema Único de Saúde (SUS).

As evidências científicas comprovam que o reconhecimento e a introdução antecipada de pré-notificação pelos serviços médicos de emergência melhoram o tratamento da doença, fazendo relação entre serviços que notificam previamente e os que os prestadores destes mesmos serviços deixam de notificar e, ainda os que chegam por outros mecanismos como em veículos particulares.

Os pesquisadores da saúde classificam as tecnologias em: 1) gerenciais - conjunto de ações teórico-práticas para administrar as ações e serviços de saúde, cujo objetivo é intervir nas práticas profissionais com a finalidade de melhorar a sua qualidade (manuais, rotinas institucionais, acolhimento e vínculo); 2) educacionais - conjunto sistemático de conhecimento científico que permite planejar, executar, controlar e acompanhar o processo educacional formal ou informal, e, assim, 
favorecer a construção e reconstrução do conhecimento (cartilhas, folhetos, vídeos); 3) tecnologias assistenciais - conjunto de saberes técnico-científicos sistematizados, processuais e instrumentais, o qual possibilita a promoção da qualidade e equipe multiprofissional da Rede de Saúde, envolvida na assistência pode prestar o cuidado ao usuário do Sistema único de Saúde SUS por meio dessas tecnologias. Portanto, a identificação do tipo de tecnologias e suas contribuições podem auxiliar a redirecionar as práticas assistenciais. Ademais, podem indicar lacunas no conhecimento quanto ao tipo de tecnologia ou pesquisa empregada e resultados referentes à temática (Maniva et al., 2018).

Segundo Portela (2017) podemos pensar a necessidade de instrumentos construídos e validados com rigor científico para avaliação de processos e resultados compõem uma estratégia para a institucionalização da avaliação em serviços de saúde.

O estudo mostra a possibilidade de inclusão de profissionais médicos, de toda e qualquer especialidade para realizar procedimentos específicos como trombólise, buscando ampliar um leque de possibilidades na saúde pública, reconhecendo nossa redução de especialistas, diferenças geográficas, vazios geográficos e assistências somados às dificuldades de reconhecimento da doença por parte da população concorrem para a redução do sucesso do tratamento.

O objetivo desta pesquisa foi de realizar uma revisão do tipo integrativa sobre ferramentas gerenciais utilizadas na Linha do Cuidado do Acidente Vascular Cerebral.

\section{Metodologia}

A classificação quanto à natureza deste estudo está pautada na pesquisa qualitativa, devido ela trabalhar os dados com a finalidade de buscar os seus significados, possuindo como base a percepção do fenômeno dentro do seu contexto (Estrela, 2018). Além disso, o estudo é também de caráter exploratório, poisas pesquisas exploratórias têm como objetivo proporcionar maior familiaridade com o problema, com vistas a torná-lo mais explícito, bem como aprimorar ideia e realizar a descoberta de intuições (Estrela, 2018).

Sendo assim, o estudo utilizou na coleta de dados tratando-se de um estudo do tipo revisão integrativa da literatura (Cunha, 2015), o qual permitiu realizar a busca, a avaliação crítica e a síntese de resultados de pesquisas sobre um tema investigado, contribuindo com o avanço do conhecimento sobre a questão norteadora deste estudo, quais os critérios de inclusão e exclusão, trazendo prioritariamente as informações que deveriam ser extraídas do estudo acerca do tema em questão com avaliação dos dados encontrados e posteriormente foi realizado a interpretação dos resultados.

Para seleção dos artigos, utilizou-se o acesso on-line em quatro bases de dados: LILACS (Latin American and Caribbean Health Sciences), MEDLINE (National Library of Medicine), SCOPUS - Base de dados multidisciplinar e CINAHL (Cumulative Index of Nursing and Allied Health Literature). Os idiomas escolhidos foram: inglês, espanhol e português com exclusão dos demais idiomas.

A pesquisa dos artigos ocorreu entre os meses de novembro de 2020 a abril de 2021, utilizando os descritores controlados em inglês "health education" e "stroke", mediados pelo operador booleano "and", visando ampliar o quantitativo de estudos. Os descritores foram extraídos do DECS (Descritores em Ciências da Saúde) do Portal BVS, do MESH (Medical Subject Headings) da National Library, e Portal de Periódicos CAPES/MEC. A questão norteadora do estudo foi: "Quais as evidências científicas que a literatura tem apontado acerca das ferramentas gerenciais para a organização da linha do cuidado do Acidente Vascular Cerebral (AVC)?". 
Quadro 1 - Descritivo da busca, recuperação e seleção de estudos sobre ferramentas gerenciais utilizadas em serviços de saúde com foco no atendimento para o Acidente Vascular Cerebral, de novembro de 2020 e abril de 2021, Belém-Pará, 2021.

\begin{tabular}{|c|c|c|c|c|}
\hline Bases de dados & $\begin{array}{c}\text { Estudos } \\
\text { selecionados } \\
\text { após a leitura } \\
\text { dos títulos }\end{array}$ & $\begin{array}{c}\text { Estudos } \\
\text { selecionados após a } \\
\text { leitura dos resumos }\end{array}$ & $\begin{array}{c}\text { Estudos } \\
\text { selecionados após a a } \\
\text { leitura na íntegra }\end{array}$ & $\begin{array}{c}\text { Estudos } \\
\text { selecionados após a } \\
\text { avaliação do rigor } \\
\text { metodológico }\end{array}$ \\
\hline LILACS & 545 & 20 & 15 & 15 \\
MEDLINE & 2692 & 28 & 10 & 04 \\
SCOPUS & 00 & 00 & 00 & 00 \\
CINAHL & 01 & 01 & 01 & 01 \\
\hline TOTAL & 3238 & 49 & 26 & 20 \\
\hline
\end{tabular}

Fonte: Busca bibliográfica dos autores (2021).

\section{Resultados}

Foram identificados 20 estudos (Quadro 1) com rigor científico, relacionados ao tema de forma que respondessem à questão norteadora do estudo no que se refere a busca do melhoramento do atendimento ao AVC e, propor a criação de ferramentas gerenciais para a linha de cuidados do AVC a partir de estudo baseado em evidências, visando aprimoramento gerencial e dos sistemas de saúde.

Descrição das etapas de seleção dos artigos incluídos na revisão integrativa. Publicações encontradas: LILACS - 545 MEDLINE - 2692, SCOPUS - 00 CINAHL - 01 TOTAL - 3238; Estudos selecionados após a leitura dos títulos: LILACS 545 MEDLINE - 2692 SCOPUS - 00 CINAHL - 01 TOTAL - 3238; Estudos selecionados após a leitura dos resumos: LILACS - 20 - MEDLINE 28 - SCOPUS 00 CINAHL - 01 TOTAL - 49; Estudos selecionados após a leitura na íntegra: LILACS - 15 MEDLINE - 10 - SCOPUS 00 CINAHL- 01 TOTAL- 26; Estudos selecionados após a avaliação do rigor metodológico: LILACS - 15 MEDLINE - 04 SCOPUS - 00 CINAHL- 01 TOTAL - 20.

A maioria dos estudos apresentou mais de uma tecnologia para o AVC (Quadro 2), as quais contemplaram as três classificações (gerenciais, educacionais e assistenciais) adotadas nesta revisão integrativa. Cuja discussão frente aos desafios foi de reconhecer a importância de novas tecnologias como aliadas ao melhoramento da assistência prestada aos pacientes acometidos por AVC adquirem um espaço cada vez maior na busca de redução de danos e melhoramento da qualidade de vida do paciente. Estes recursos são considerados práticos e com inúmeros benefícios aos usuários do SUS. Podem também contribuir para o aumento de conhecimento para os profissionais de saúde, acesso rápido e melhores prognósticos aos usuários do SUS.

A importância da terapia trombolítica para AVC isquêmico vem ganhando destaque sendo evidenciada neste contexto, onde é realizada uma pesquisa para elucidar se esta prática possui segurança e uma possível lacuna na relação entre protocolos assistenciais atuais e o tempo-resposta para atingir o tratamento.

Segundo estudo de Haverkamp (2018) que a trombólise está sendo fornecida de forma eficaz e segura em serviços de saúde na Irlanda, apesar do envolvimento de equipe não especializada. Neste sentido podemos pensar na inclusão de profissionais médicos, de toda e qualquer especialidade para realizar esse procedimento, buscando ampliar um leque de possibilidades na saúde pública, reconhecendo nossa redução de especialistas, diferenças geográficas e assistências somados às dificuldades de reconhecimento da doença por parte da população concorrem para a redução do sucesso do tratamento. 


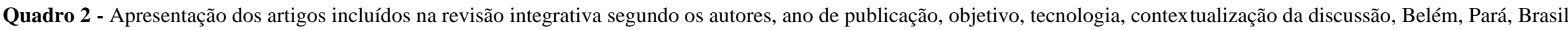
2021.

\begin{tabular}{|c|c|c|c|c|c|}
\hline $\mathbf{N}^{\mathbf{o}}$ & Título & Autores & Objetivo & Tecnologia & Contextualização da discussão \\
\hline 1 & $\begin{array}{l}\text { Tecnologias educacionais, assistenciais e } \\
\text { gerenciais: uma reflexão a partir da concepção } \\
\text { dos docentes de enfermagem. }\end{array}$ & $\begin{array}{l}\text { Nietsche et al } \\
\text { (2005); } \\
\text { Brasil. }\end{array}$ & $\begin{array}{l}\text { Identificar as tecnologias } \\
\text { assistenciais, educacionais e } \\
\text { gerenciais que vêm sendo } \\
\text { utilizadas e/ou produzidas em } \\
\text { suas práxis profissionais nas } \\
\text { disciplinas profissionalizantes. }\end{array}$ & $\begin{array}{l}\text { Instrumento de } \\
\text { construído a partir da } \\
\text { pesquisa-ação. }\end{array}$ & $\begin{array}{l}\text { Salienta-se, portanto, que a temática tecnologia, enquanto } \\
\text { processo e/ou produto, necessita ser discutida, repensada, } \\
\text { estudada e construída, pois ainda se encontra incipientemente } \\
\text { abordada nas práxis dos profissionais de enfermagem. }\end{array}$ \\
\hline 2 & $\begin{array}{l}\text { Addressing stroke signs and symptoms through } \\
\text { public education: the stroke heroes act fast } \\
\text { campaign. }\end{array}$ & $\begin{array}{l}\text { Wall et al } \\
\text { (2008); EUA. }\end{array}$ & $\begin{array}{l}\text { O objetivo do estudo qualitativo } \\
\text { e explorar a necessidade de } \\
\text { suporte do cuidador ao longo do } \\
\text { tempo. }\end{array}$ & Capacitação & $\begin{array}{l}\text { A falta de qualificação dos cuidadores pode ser considerada } \\
\text { um fator importante para o sucesso da continuidade dos } \\
\text { pacientes pós evento de AVC. }\end{array}$ \\
\hline 3 & $\begin{array}{l}\text { A oferta de tomógrafo computadorizado para o } \\
\text { tratamento do Acidente Vascular Cerebral } \\
\text { agudo, no Brasil, sob o ponto de vista das } \\
\text { desigualdades sociais e geográficas. }\end{array}$ & $\begin{array}{l}\text { Gutierrez } \\
\text { (2009); } \\
\text { Brasil. }\end{array}$ & $\begin{array}{l}\text { Realização do diagnóstico em } \\
\text { tempo Hábil. Diminuição das } \\
\text { desigualdades sociais através de } \\
\text { tecnologia. }\end{array}$ & $\begin{array}{l}\text { Tomógrafos } \\
\text { computadorizados. }\end{array}$ & $\begin{array}{l}\text { Mostram uma distribuição geográfica de TC pouco } \\
\text { equitativa, e uma concentração da oferta dessa tecnologia em } \\
\text { municípios mais populosos e de melhor situação } \\
\text { socioeconômica. }\end{array}$ \\
\hline 4 & $\begin{array}{l}\text { Creating a supportive environment for living } \\
\text { with stroke in rural areas: two low-cost } \\
\text { community-based interventions. }\end{array}$ & $\begin{array}{l}\text { Newell et al } \\
\text { (2009); EUA. }\end{array}$ & $\begin{array}{l}\text { Apoio e reintegração à } \\
\text { comunidade rural dos pacientes e } \\
\text { cuidadores do AVC na Nova } \\
\text { Escócia Canadá. }\end{array}$ & $\begin{array}{c}\text { Guia de recursos } \\
\text { informativos para pessoas } \\
\text { que vivem em } \\
\text { comunidade rural, e } \\
\text { apoio emocional. }\end{array}$ & $\begin{array}{l}\text { Este estudo não mostra resultados significativos, pois precisa } \\
\text { de continuidade. }\end{array}$ \\
\hline 5 & $\begin{array}{c}\text { Knowledge of Risk Factores and Warning } \\
\text { Signs of Stroke: A Systematic Review from a } \\
\text { Gender Perspective. }\end{array}$ & $\begin{array}{l}\text { Stroebele } \\
\text { et al (2011); } \\
\text { Alemanha. }\end{array}$ & $\begin{array}{l}\text { Avaliar o conhecimento sobre os } \\
\text { fatores de risco e os sinais de } \\
\text { alerta na população em geral. }\end{array}$ & Revisão sistemática. & $\begin{array}{l}\text { No geral foi observado melhor conhecimento sobre o AVC } \\
\text { em mulheres comparando com homens embora haja falta de } \\
\text { conhecimento em ambos os sexos. }\end{array}$ \\
\hline 6 & $\begin{array}{l}\text { Acesso, prática educativa e empoderamento de } \\
\text { pacientes com doenças crônicas. }\end{array}$ & $\begin{array}{l}\text { Silva et al } \\
\text { (2012); } \\
\text { Brasil. }\end{array}$ & $\begin{array}{l}\text { Avaliar o papel que o profissional } \\
\text { de saúde desempenha no } \\
\text { empoderamento do usuário para } \\
\text { um cuidado promotor de saúde }\end{array}$ & $\begin{array}{l}\text { Práticas de educação } \\
\text { permanente em saúde. }\end{array}$ & $\begin{array}{c}\text { A educação tem o papel de formar consciência crítica e a } \\
\text { autonomia, necessitando de escuta ativa e o diálogo aberto e } \\
\text { incentivar as pessoas a ter autonomia para entendimento e } \\
\text { formação das ações. }\end{array}$ \\
\hline 7 & $\begin{array}{l}\text { Stroke communication: findings from Hip Hop } \\
\text { Stroke. }\end{array}$ & $\begin{array}{l}\text { Williams et } \\
\text { al; (2012); } \\
\text { EUA. }\end{array}$ & $\begin{array}{l}\text { Avaliar se o atraso para o uso de } \\
\text { trombolíticos está associada a } \\
\text { baixa comunicação do público } \\
\text { em geral sobre AVC. }\end{array}$ & $\begin{array}{c}\text { Alfabetização de crianças } \\
\text { em escolas e informação } \\
\text { sobre o AVC. }\end{array}$ & $\begin{array}{l}\text { Discussão sobre programas de alfabetização de pais de } \\
\text { crianças poderiam melhorar conhecimentos sobre o AVC. }\end{array}$ \\
\hline
\end{tabular}


Research, Society and Development, v. 10, n. 7, e11110716262, 2021

(CC BY 4.0) | ISSN 2525-3409 | DOI: http://dx.doi.org/10.33448/rsd-v10i7.16262

\begin{tabular}{|c|c|c|c|c|c|}
\hline 8 & $\begin{array}{l}\text { Evaluation of patients with stroke monitored } \\
\text { by home care programs }\end{array}$ & $\begin{array}{l}\text { Oliveira et al } \\
\text { (2013); } \\
\text { Brasil. }\end{array}$ & $\begin{array}{c}\text { Avaliar a capacidade de } \\
\text { identificar o AVC no pré e pós } \\
\text { evento. }\end{array}$ & $\begin{array}{l}\text { Distribuição de folhetos } \\
\text { em áreas específicas. }\end{array}$ & $\begin{array}{c}\text { Adaptação de programas para subgrupos especiais como } \\
\text { parentes de pessoas com AVC. }\end{array}$ \\
\hline 9 & $\begin{array}{l}\text { Effects of stroke education using an animated } \\
\text { cartoon and a manga on elementary school } \\
\text { children. }\end{array}$ & $\begin{array}{l}\text { Sakamoto et } \\
\text { al (2014); } \\
\text { EUA. }\end{array}$ & $\begin{array}{l}\text { Avaliar a viabilidade e eficácia } \\
\text { dos materiais sobre o AVC. }\end{array}$ & $\begin{array}{l}\text { Desenho animado e um } \\
\text { mangá para alunos do } \\
\text { ensino Médio. }\end{array}$ & $\begin{array}{c}\text { Espera-se que a informação sobre o AVC para Jovens reduza } \\
\text { o atraso pré-hospitalar. }\end{array}$ \\
\hline 10 & $\begin{array}{l}\text { Improving adherence to secondary stroke } \\
\text { prevention strategies through motivational } \\
\text { interviewing: a randomised controlled trial. }\end{array}$ & $\begin{array}{l}\text { Barker-Collo } \\
\text { et al; (2015); } \\
\text { EUA. }\end{array}$ & $\begin{array}{l}\text { Melhoria da adesão ao tratamento } \\
\text { medicamentoso e importância da } \\
\text { mudança de estilo de vida. }\end{array}$ & $\begin{array}{l}\text { Entrevista motivacional } \\
\text { aos pacientes. }\end{array}$ & $\begin{array}{l}\text { Aulas específicas sobre o AVC são exigidas como parte } \\
\text { curricular dos Enfermeiros para o reconhecimento precoce do } \\
\text { AVC agudo, e na oportunidade nos mostra a importância da } \\
\text { atenção psicossocial. }\end{array}$ \\
\hline 11 & $\begin{array}{l}\text { Stroke Education in an Emergency Department } \\
\text { Waiting Room: A Comparison of Methods. }\end{array}$ & $\begin{array}{c}\text { Chan et al } \\
\text { (2015); EUA. }\end{array}$ & $\begin{array}{l}\text { Avaliar a eficiência e eficácia das } \\
\text { tecnologias individuais, e em } \\
\text { conjunto para reconhecer sinais e } \\
\text { sintomas de AVC }\end{array}$ & $\begin{array}{l}\text { Vídeo, aconselhamento } \\
\text { individual e folheto com } \\
\text { informações sobre o } \\
\text { AVC. }\end{array}$ & $\begin{array}{l}\text { Cada método falhou em um aumento duradouro do } \\
\text { conhecimento quando avaliado separadamente }\end{array}$ \\
\hline 12 & $\begin{array}{l}\text { Using visualization to explore temporal } \\
\text { multidimensional data of stroke patients. }\end{array}$ & $\begin{array}{l}\text { Loorak, et al } \\
\text { (2016). EUA. }\end{array}$ & $\begin{array}{c}\text { Impacto dos procedimentos, } \\
\text { eventos e atributos do paciente } \\
\text { no tempo, para salvar vidas e } \\
\text { melhorar a qualidade de vida } \\
\text { após o AVC. }\end{array}$ & $\begin{array}{l}\text { TimeSpan, uma } \\
\text { ferramenta baseada na } \\
\text { web, para explorar dados } \\
\text { multidimensionais. }\end{array}$ & $\begin{array}{c}\text { Ferramenta baseada na web para explorar dados e traços. Para } \\
\text { análise crítica dos eventos e atributos dos pacientes } \\
\text { relacionadas ao tempo para salvar vidas e melhorar a } \\
\text { qualidade de vida no AVC. }\end{array}$ \\
\hline 13 & $\begin{array}{c}\text { Delivering knowledge of stroke to parents } \\
\text { through their children using a manga for stroke } \\
\text { education in elementary school. J Stroke } \\
\text { Cerebrovas. }\end{array}$ & $\begin{array}{l}\text { Ishigami et al } \\
\text { (2017); EUA. }\end{array}$ & $\begin{array}{l}\text { Esclarecer a eficácia de novos } \\
\text { recursos educacionais para } \\
\text { crianças e seus pais do ensino } \\
\text { fundamental. }\end{array}$ & $\begin{array}{l}\text { Palestras em escolas com } \\
\text { ou sem neurologista. }\end{array}$ & $\begin{array}{c}\text { A escola seria promissora para divulgar amplamente o } \\
\text { conhecimento sobre o AVC. }\end{array}$ \\
\hline 14 & $\begin{array}{l}\text { Feasibility and utility of an integrated medical } \\
\text { imaging and informatics smartphone system } \\
\text { for management of acute stroke. }\end{array}$ & $\begin{array}{l}\text { Shikirkova et } \\
\text { al (2017); } \\
\text { EUA. }\end{array}$ & Valor para o tratamento. & $\begin{array}{l}\text { Sistema informativo } \\
\text { móvel } \\
\text { (Syinapse ERm). }\end{array}$ & $\begin{array}{c}\text { A Synapse ERm foi considerada viável de utilizar com } \\
\text { integração de dados clínicos e de imagem, e moderada } \\
\text { utilidade no gerenciamento terapêutico. }\end{array}$ \\
\hline 15 & $\begin{array}{c}\text { Construção e validação de instrumento para } \\
\text { avaliação da qualidade dos processos e } \\
\text { resultados do serviço de atendimento móvel de } \\
\text { urgência relacionada ao acidente vascular } \\
\text { cerebral. }\end{array}$ & $\begin{array}{l}\text { Portela } \\
(2017) ; \\
\text { Brasil. }\end{array}$ & $\begin{array}{l}\text { Construir e validar um } \\
\text { instrumento para avaliação dos } \\
\text { serviços móveis de urgência - } \\
\text { SAMU. }\end{array}$ & $\begin{array}{l}\text { Tomada de decisão } \\
\text { gerencial e } \\
\text { aprimoramento dos } \\
\text { sistemas de saúde. }\end{array}$ & $\begin{array}{l}\text { Busca avaliar a qualidade dos processos de trabalho e } \\
\text { resultado dos serviços do Atendimento Móvel de Urgência } \\
\text { (SAMU) para o AVC. }\end{array}$ \\
\hline 16 & $\begin{array}{l}\text { Network-based approach to identify potential } \\
\text { targets and drugs that promote neuroprotection } \\
\text { and neurorepair in acute ischemic stroke. }\end{array}$ & $\begin{array}{l}\text { Wang et al } \\
\text { (2017); } \\
\text { China. }\end{array}$ & $\begin{array}{l}\text { Rápida disseminação e } \\
\text { coordenação de dados clínicos. }\end{array}$ & $\begin{array}{l}\text { Estudo de associação } \\
\text { Genética, Humanos. }\end{array}$ & $\begin{array}{l}\text { O AVC Isquêmico é responsável por mais de } 80 \% \text { dos } \\
\text { aproximadamente } 610.000 \text { novos casos no mundo. Tanto a } \\
\text { isquemia quanto a reperfursão podem causar a morte. As } \\
\text { terapias genéticas como solução ainda se encontram em fase }\end{array}$ \\
\hline
\end{tabular}


Research, Society and Development, v. 10, n. 7, e11110716262, 2021

(CC BY 4.0) | ISSN 2525-3409 | DOI: http://dx.doi.org/10.33448/rsd-v10i7.16262

\begin{tabular}{|c|c|c|c|c|c|}
\hline & & & & & experimental. \\
\hline 17 & $\begin{array}{l}\text { Regional differences in thrombectomy rates: } \\
\text { secondary use of billing codes in the miracum } \\
\text { (medical informatics for research and care in } \\
\text { university medicine). }\end{array}$ & $\begin{array}{l}\text { Haverkamp et } \\
\text { al; (2018); } \\
\text { EUA. }\end{array}$ & $\begin{array}{l}\text { Avaliação do uso de } \\
\text { trombolíticos nos centros de } \\
\text { Neurologia sem variável fixa, } \\
\text { como: idade, sexo ou dia da } \\
\text { semana. }\end{array}$ & $\begin{array}{l}\text { Instrumento para } \\
\text { avaliação de dados. }\end{array}$ & $\begin{array}{l}\text { A trombectomia mecânica, além da trombólise mecânica são } \\
\text { recomendadas para o tratamento de AVC agudo em pacientes } \\
\text { com oclusão de grandes vasos. }\end{array}$ \\
\hline 18 & $\begin{array}{l}\text { Clinical information systems integration in } \\
\text { new york city's first mobile stroke unit. }\end{array}$ & $\begin{array}{l}\text { Kummer, BR. } \\
\text { et al (2018); } \\
\text { EUA. }\end{array}$ & $\begin{array}{l}\text { Redução do tempo de terapia } \\
\text { trombolítica para AVC. }\end{array}$ & $\begin{array}{l}\text { Desenvolvimento de } \\
\text { aplicativo conectado à } \\
\text { rede hospitalar para } \\
\text { entrada de dados do } \\
\text { paciente. }\end{array}$ & $\begin{array}{l}\text { Ocorreram duas falhas de rede a bordo, resultando em mau } \\
\text { funcionamento do scanner de tomografia computadorizada, } \\
\text { porém nenhum paciente se tornou inelegível para o } \\
\text { tratamento. }\end{array}$ \\
\hline 19 & $\begin{array}{l}\text { Tecnologias educativas para o acidente } \\
\text { vascular cerebral: revisão integrativa. }\end{array}$ & $\begin{array}{l}\text { Maniva et al } \\
\quad \text { (2018); } \\
\text { Brasil. }\end{array}$ & $\begin{array}{l}\text { Identificar na literatura científica } \\
\text { as tecnologias educativas } \\
\text { utilizadas no processo de } \\
\text { educação em saúde relacionadas } \\
\text { ao AVC. }\end{array}$ & Tecnologias educativas. & $\begin{array}{l}\text { As tecnologias educativas se mostraram necessárias e } \\
\text { relevantes, e as informações melhoram o conhecimento e o } \\
\text { enfrentamento do paciente. }\end{array}$ \\
\hline 20 & $\begin{array}{l}\text { Integration of real-time electronic health } \\
\text { records and wireless technology in a mobile } \\
\text { stroke unit. }\end{array}$ & $\begin{array}{l}\text { Schimpf et al } \\
\text { (2019); EUA. }\end{array}$ & $\begin{array}{c}\text { Projetar infraestrutura de } \\
\text { tecnologia da informação única e } \\
\text { dinâmica. }\end{array}$ & $\begin{array}{l}\text { Scanner de tomógrafo e } \\
\text { telemedicina. }\end{array}$ & $\begin{array}{l}\text { As imagens do cérebro adquiridas no scanner de TC da } \\
\text { ambulância são transferidas inicialmente para um laptop de } \\
\text { bordo. } \\
\text { Buscam melhorar a qualidade dos serviços móveis de } \\
\text { urgência. }\end{array}$ \\
\hline
\end{tabular}

Fonte: Busca bibliográfica dos autores (2021). 


\section{Discussões}

No atual quadro epidemiológico da região do estado do Pará prevalecem as DCNT (doenças crônicas não transmissíveis) em destaque as doenças cardiovasculares, juntamente as de causas externas, resultam na falta de acesso a serviços de saúde qualificados. As condições socioeconômicas, ambientais, e estruturais desfavoráveis, da maioria dos municípios, têm contribuído para a manutenção de cenários epidemiológicos onde coexistem as doenças ditas "da pobreza" (Pará, 2015).

A revisão bibliográfica realizada avaliou as tecnologias utilizadas no cuidado ao paciente com AVC, as quais tem significativa relevância no fortalecimento da Rede de Urgência e Emergência, e constituindo-se em diretrizes a resolutividade e o acesso aos serviços de saúde com integralidade, e de forma rápida para oportunizar qualidade ao atendimento.

Neste contexto foram avaliadas tecnologias inovadoras e tecnologias de educação. As tecnologias de educação mostraram considerada relevância, tanto no enfrentamento do paciente para as questões de identificação precoce dos primeiros sinais, quanto para orientação de cuidadores para continuidade do tratamento, bem como a doença durante a internação, e essas práticas podem proporcionar autonomia melhorando a autonomia dos pacientes.

Observa-se um destaque para a Atenção Primária à Saúde no que se refere às tecnologias Leve e duras, onde há um predomínio de tecnologias voltadas para a educação, tais como: palestras, cartilhas, e informativos todos voltados ao esclarecimento de pacientes de sinais e sintomas relacionados à doença. Embora seja considerado de grande importância o uso de folhetos e cartilhas, não houve a participação dos profissionais de saúde na confecção deste material permitiu observar que a metodologia participativa e comunicativa não tem sido utilizada no processo de elaboração desses materiais.

Algumas tecnologias foram aplicadas para avaliar o nível de conhecimento no paciente, bem como na população visando estabelecer o grau de conhecimento, e avaliar o tempo de duração das informações. As condições socioeconômicas e geográficas são referenciadas como um fator desfavorável para o acesso ao tratamento, e para as informações onde pacientes em zonas rurais necessitam de tecnologias de apoio e diferenciadas para a prevenção, e continuidade do tratamento.

Diante desse cenário podemos constatar que tais condições corroboram com o atual quadro epidemiológico da região do estado, onde prevalecem as DNTC (doenças não transmissíveis crônicas) em destaque as doenças cardiovasculares, juntamente as de causas externas, as derivadas de acidentes e agressões, e que as mesmas resultam na falta de acesso a serviços de saúde qualificados." As condições socioeconômicas, ambientais, e estruturais desfavoráveis, da maioria dos municípios do Pará, têm contribuído para a manutenção de cenários epidemiológicos onde coexistem as doenças ditas "da pobreza" (Pará, 2015).

Para avaliar a linha de cuidados do AVC é necessário conhecer as tecnologias utilizadas para a comunicação dos profissionais em suas rotinas dos serviços de saúde, suas próprias experiências desenvolvidas ao longo do tempo, qualificação profissional para o enfrentamento, infraestrutura do local para atendimento eficiente e eficaz até as práticas e modelos de atendimento utilizados em prol do paciente. Para isso, cabe analisar a estrutura compreendida para o recebimento e tratamento dos pacientes.

Devemos destacar a importância de novas tecnologias para o enfrentamento do AVC. Podemos constatar a importância do reconhecimento dos sinais e sintomas precocemente e a orientação em protocolos para acionar imediatamente os serviços médicos de urgência, como encontrado no código do AVC em serviços hospitalares, e como suporte na orientação da população em geral, e na Atenção Primária à Saúde.

A disseminação de dados clínicos e de imagem entre membros da equipe multidisciplinar, tem como objetivo viabilizar o sistema informático móvel, tomografia computadorizada, ressonância magnética e imagem angiográfica de cateter 
com comunicação em tempo real; bancos de dados no hospital de referência onde todos tenham acesso às informações dos pacientes, através de intranet e aplicativos clínicos (Kummer et al., 2018).

Loorak (2016) apresentou em seu estudo o TimeSpan, ferramenta de visualização exploratória que com base nas tarefas e no entendimento gera uma colaboração projetada em tempo real na Web”. Já Schimpf (2019) destacou a unidade Móvel de AVC da UCHealth (MSU) no hospital da universidade do colorado, ambulância equipada com Scanner de Tomografia computadorizada.

A comunicação utilizada entre os profissionais de saúde, suas habilidades e dificuldades no processo do cuidar em todos os níveis de atenção à saúde foi observada como de significativa importância.

Outro aspecto percebido nesta revisão integrativa foi o fato da maioria dos trabalhos sobre tecnologias para a linha de cuidados do AVC possuir um modelo de pesquisa teórico, ou, quando empírico, de bases quantitativas, sendo encontrados poucos estudos da realidade sob uma ótica qualitativa. Entende-se que essa é uma fraqueza da teoria, pois, muito embora os resultados de vários dos trabalhos empíricos tenham sido estatisticamente relevantes, há uma rica coleta de dados vivenciais.

Podemos perceber ainda o predomínio de tecnologias do tipo assistenciais interligadas às educacionais na maioria dos estudos, considerando que uma boa parte das tecnologias avançadas foram encontradas fora de nosso país, o que mostra o avanço tecnológico em países desenvolvidos, os quais através de pesquisas avançadas possuem os melhores resultados para assistir de forma qualificada seus pacientes.

\section{Considerações Finais}

A proposta da busca de uma ferramenta gerencial a partir da revisão integrativa visou avaliar a lacuna da atual comunicação na linha de cuidados do AVC. Os resultados obtidos por meio dos estudos selecionados nesta revisão integrativa da literatura fornecem evidências de que a linha de cuidado precisa ser melhor discutida entre os profissionais da saúde e a sociedade. Sabemos que é importante que os pacientes acometidos por doenças cerebrovasculares sejam assistidos por uma equipe multiprofissional capacitada, onde os profissionais possam acompanhar o impacto de suas intervenções por meio da avaliação contínua, pois as sequelas do AVC causam limitações e mudanças na qualidade de vida do paciente. Assim fica evidente que para um atendimento eficiente é imprescindível que o assunto seja discutido dentro da academia e haja qualificação dos profissionais de saúde.

Portanto, para trabalhos futuros, será necessário a atualização desta revisão integrativa de literatura com a finalidade de implementação para possíveis pesquisas de estudiosos que se interessem por pesquisar esta temática. Assim, podemos concluir ratificando que é possível melhorar a qualidade de vida dos pacientes acometidos de AVC por meio da existência de ferramentas de tecnologias gerenciais que garantam de forma rápida e efetiva a comunicação entre a equipe multidisciplinar e a gerência dos serviços de saúde. Entretanto, se faz necessário o investimento em novas pesquisas que possibilitem um maior conhecimento sobre as dificuldades enfrentadas pelas equipes multidisciplinares e a gerência dos serviços de saúde.

\section{Referências}

Barker-Collo, S., Krishnamurthi, R., Witt, E., Feigin, V., Jones, A., Mcpherson, K., Starkey, N., Parag, V., Jiang, Y., Barber, P. A., Rush, E., Bennett, D., \& Aroll, B. (2015). Improving adherence to secondary stroke prevention strategies through motivational interviewing: Randomized controlled trial. Stroke, 46(12), 3451-3458.

Chan, Y.-F. Y., Richardson, L. D., Nagurka, R., Hao, K., Zaets, S. B., Brimacombe, M. B., Bentley, S., \& Levine, S. R. (2015). Stroke Education in an Emergency Department Waiting Room: a Comparison of Methods. Health Promotion Perspectives, 5(1), 34-41.

Cunha, M. C. (2015). Literature review: A review with a focus on the systematic reviews. Codas, 27(5), 409-410.

Estrela, C. (2018). Metodologia científica: ciência, ensino, pesquisa. Artes Médicas. 
Gutierrez, M. . (2009). A Oferta de Tomógrafo Computadorizado para o Tratamento do Acidente Vascular Cerebral Agudo, no Brasil, sob o Ponto de Vista das Desigualdades Sociais e Geográficas. Fundação Oswaldo Cruz, Escola Nacional de Saúde Pública Sergio Arouca.

Haverkamp, C., Ganslandt, T., Horki, P., \& Boeker, M. (2018). Regional Differences in Thrombectomy Rates: Secondary use of Billing Codes in the MIRACUM (Medical Informatics for Research and Care in University Medicine) Consortium. Clinical Neurology, $28(2), 225-234$.

Ishigami, A., Yokota, C., Nishimura, K., Ohyama, S., Tomari, S., Hino, T., Arimizu, T., Wada, S., Toyoda, K., \& Minematsu, K. (2017). Delivering Knowledge of Stroke to Parents Through Their Children Using a Manga for Stroke Education in Elementary School. Journal of Stronke and Cerebrovascular Diseases, 26(2), 431-437.

Kummer, B. R., Lerario, M. P., Navi, B. B., Ganzman, A. C., Ribaudo, D., Mir, S. A., Pishanidar, S., Lekic, T., Williams, O., Kamel, H., Marshall, R. S., Hripcsak, G., Elkind, M. S. V., \& Fink, M. E. (2018). Clinical Information Systems Integration in New York City's First Mobile Stroke Unit. Applied Clinical Informatics, 9(1), 89-98.

Loorak, M. H., Perin, C., Kamal, N., Hill, M., \& Carpendale, S. (2016). TimeSpan: Using Visualization to Explore Temporal Multi-dimensional Data of Stroke Patients. IEEE Transactions on Visualization and Computer Graphics, 22(1), 409-418.

Maniva, S. J. C. de F., Carvalho, Z. M. de F., Gomes, R. K. G., Carvalho, R. E. F. L. de, Ximenes, L. B., \& Freitas, C. H. A. de. (2018). Educational technologies for health education on stroke: an integrative review. Revista Brasileira de Enfermagem, 71(suppl 4), $1724-1731$.

Newell, J. M., Lyons, R., Martin-Misener, R., \& Shearer, C. L. (2009). Creating a supportive environment for living with stroke in rural areas: two low-cost community-based interventions. Topics in Stroke Rehabilitation, 16(2), 147-156.

Nietsche, E. A., Backes, V. M. S., Colomé, C. L. M., Ceratti, R. do N., \& Ferraz, F. (2005). Tecnologias Educacionais, Assistenciais E Gerenciais: Uma Reflexão A Partir Da Concepção Dos Docentes De Enfermagem. Revista Latino Americana De Enfermagem, 13(3), $344-353$.

Oliveira, A. R. de S., de Araujo, T. L., de Sousa Costa, A. G., Morais, H. C. C., da Silva, V. M., \& de Oliveira Lopes, M. V. (2013). Evaluation of patients with stroke monitored by home care programs. Revista Da Escola de Enfermagem, 47(5), 1143-1149.

Pará. (2015). Plano estadual de atenção integral às urgências (p. 98). SESPA.

Portela, F. (2017). Construção e validação de instrumento para avaliação da qualidade dos processos e resultados do serviço de atendimento móvel de urgência relacionadas ao acidente vascular cerebral. Universidade Federal do Paraná.

Sakamoto, Y., Yokota, C., Fumio, M., \& Amano, T. (2014). No Effects of stroke education using an animated cartoon and a manga on elementary school children. J Stroke Cerebrovasc Dis, 23(7), 1877-1881.

Schimpf, B., Deanda, K., Severenuk, D. A., Montgomery, T. M., Cooley, G. D., Kowalski, R. G., Vela-Duarte, D., \& Jones, W. J. (2019). Integration of RealTime Electronic Health Records and Wireless Technology in a Mobile Stroke Unit. J Stroke Cerebrovasc Dis, 28(9), $2530-2536$.

Shkirkova, K., Akam, E. Y., Huang, J., Sheth, S. A., Nour, M., Liang, C. W., Mcmanus, M., Trinh, V., Duckwiler, G., Tarpley, J., Vinuela, F., \& Saver, J. L. (2017). Feasibility and utility of an integrated medical imaging and informatics smartphone system for management of acute stroke. International Journal of Stroke, 12(9).

Stroebele, N., Müller-riemenschneider, F., Nolte, C. H., \& Müller-Nordborn, J. (2011). Knowledge of risk factors, and warning signs of stroke: a systematic review from a gender perspective. International Journal of Stroke, 6(1), 60-65.

Taddeo, P. da S., Gomes, K. W. L., Caprara, A., de Amorim Gomes, A. M., de Oliveira, G. C., \& Maria, T. M. M. (2012). Acesso, prática educativa e empoderamento de pacientes com doenças crônicas. Ciencia e Saude Coletiva, 17(11), 2923-2930.

Wall, H. K., Beagan, B. M., June ONeill, H., Foell, K. M., Boddie-Willis, C. L., \& for CDC, M. (2008). Addressing Stroke Signs and Symptoms Through Public Education: The Stroke Heroes Act FAST Campaign. Public, Health Reasearch, Practice, and Policy, 5(2), 1-10.

Wang, Y., Liu, H., Lin, Y., Liu, G., Chu, H., Zhao, P., Yang, X., Zheng, T., Fan, M., Zhou, X., Meng, J., \& Sun, C. (2017). Network-Based Approach to Identify Potential Targets and Drugs that Promote Neuroprotection and Neurorepair in Acute Ischemic Stroke. Scientific Reports, 2 (7), 55.

Williams, O., Desorbo, A., Noble, J., \& Gerin, W. (2012). Child-mediated stroke communication: Findings from Hip Hop stroke. Stroke, 43(1), 163-169. 